\title{
Os Benefícios da Natação Adaptada em Indivíduos com Lesões Neurológicas
}

\author{
Olívia Tsutsumi ${ }^{1}$, Viviane da Silva Cruz ${ }^{2}$, Berenice Chiarello ${ }^{3}$, Domingos Belasco Junior ${ }^{4}$, \\ Sandra Regina Alouche 5
}

\section{RESUMO}

A prática de esportes para os deficientes físicos representa a aplicação de filosofia e de princípios de reabilitação no mais alto nível. A natação é um dos esportes mais completos e proporciona uma variedade de benefícios tanto para indivíduos em geral como para os portadores de algum tipo de deficiência física. O objetivo deste estudo é, por meio de revisão bibliográfica, apresentar os benefícios que a natação adaptada pode proporcionar aos indivíduos portadores de lesões neurológicas com seqüelas motoras.

Unitermos: Esporte adaptado, Natação.

\section{SUMMARY}

Sports for physically handicapped represent an application of the philosophy and the principles of rehabilitation on the highest level. Swimming is one of the most complete sports and provides a variability of benefits for both individuals in general and those with any kind of disability. The purpose of this study is, through a bibliographic revision, to present the benefits that adapted swimming provides to individuals with neurological diseases with motor sequels.

Keywords: Adapted sport, Swimming.

\section{INTRODUÇÃO}

A cada dia, um número maior de pessoas com algum tipo de incapacidade física está envolvida em atividades físicas e esportes devido aos benefícios que eles trazem para a reabilitação e para o bem - estar ${ }^{1}$. A exclusão desses indivíduos da prática de atividade física ou do esporte pode levar a diminuição da aptidão física, da eficiência dos movimentos ou mesmo do desenvolvimento de habilidades motoras ${ }^{2}$.

A natação é um dos esportes mais apropriados para indivíduos com algum tipo de deficiência física, devido aos benefícios e às facilidades proporcionados pela execução de movimentos com o corpo imerso na água. A natação desenvolve coordenação, condicionamento aeróbio, reduz a espasticidade, e resulta em menos fadiga que outras atividades. Além disso, traz grandes contribuições para o processo de reabilitação e pode reduzir o grau de fraqueza e de complicações ${ }^{1}$. Para pessoas com deficiência, a natação tem valor terapêutico, recreativo e também social ${ }^{3}$.
O objetivo deste estudo é apresentar os benefícios que a natação adaptada pode proporcionar aos indivíduos portadores de lesões neurológicas com alguma incapacidade física.

\section{MÉTODO}

Revisão bibliográfica da literatura publicada sobre esportes adaptados e natação adaptada, no período de 1979 2003, usando as bases de dados lilacs, medline, embase; em inglês e português.

\section{Programa de reabilitação e o esporte}

Para um programa de reabilitação efetivo é importante o trabalho de uma equipe multiprofissional além da integração familiar e da sociedade onde o indivíduo vive ${ }^{4}$. Os objetivos de um programa de reabilitação enfocam a 
independência nas atividades de vida diária e nas atividades profissionais. É essencial considerar que o indivíduo, neste processo de reabilitação, necessita também de recreação e de atividade esportiva como qualquer outro ${ }^{2}$.

RIMMER (1999) sugere quatro componentes na definição da promoção da saúde para indivíduos com deficiência: promoção de estilo de vida saudável e meio ambiente saudável; prevenção de complicações de saúde (condições médicas secundárias) e outras complicações da deficiência; preparação da pessoa com deficiência para compreender e monitorar a própria saúde e necessidades de cuidados especiais; promoção de oportunidades para participação em atividades diárias comuns ${ }^{5}$.

O esporte adaptado foi definido por WINNICK (1990) como sendo "experiências esportivas modificadas ou especificamente designadas para suprir as necessidades especiais de indivíduos. O árbitro do esporte adaptado inclui a interação e os lugares nos quais se incluem apenas pessoas com condições de deficiência" 6 .

O esporte adaptado é indicado desde a fase inicial do processo de reabilitação. Os indivíduos têm a oportunidade de vivenciar sensações e movimentos, que muitas vezes não realizaram pela limitação física ou por barreiras sociais e ambientais ${ }^{7}$.

\section{Histórico do esporte adaptado}

O esporte para portadores de deficiência física iniciou na Inglaterra e nos EUA com a reabilitação de veteranos da II Guerra Mundial 4,8. Em 1944, Sir Ludwig Guttmann, neurologista e neurocirurgião alemão, foi convidado pelo governo britânico a dirigir um centro de lesão medular, no Hospital de Stoke Mandeville 4,9,10. Ele introduziu algumas modalidades esportivas, inicialmente com arco e flecha e o pólo em cadeira de rodas e, posteriormente, o tênis de mesa, a sinuca, a natação e o basquete em cadeira de rodas. A primeira competição realizada foi com basquete em cadeia de rodas e a partir daí, o centro foi palco de competições nacionais e internacionais ${ }^{4}$.

O basquete em cadeira de rodas foi bastante desenvolvido nos EUA com um caráter altamente competitivo. Em 1945, um grupo de administração hospitalar de veteranos da guerra organizou o $1^{\circ}$ campeonato sobre a direção dos Veteranos Paralisados da América (PVA) ${ }^{4}$.

A Associação Nacional de Basquete em Cadeira de Rodas (NWBA) foi formada em 1949, dando um novo rumo ao desenvolvimento dos esportes adaptados ${ }^{4}$.

Em 1952, os jogos de Stoke Mandeville tornaram-se internacionais com a entrada de um grupo da Holanda nas competições. Passaram então a se chamar Jogos Internacionais de Stoke Mandeville (ISMG) e foi formada a Federação Internacional dos Jogos de Stoke Mandeville (ISMGF). Em 1957, Benjamim H. Liptom introduziu outras modalidades esportivas sobre cadeira de rodas nos EUA, que até então era somente voltada ao basquete. No ano seguinte, surgiram oportunidades para outros esportes nos campeonatos americanos, como atletismo, natação, tênis de mesa e arco e flecha, devido à formação da Associação do Atletismo em Cadeira de Rodas (NWAA) ${ }^{4}$.
Em 1960, houve uma integração da NWAA com o corpo internacional do movimento, que passou a se chamar Comitê Internacional dos Jogos de Stoke Mandeville (ISMGC) ${ }^{4}$. Neste mesmo ano, ocorreu a primeira paraolimpíada, em Roma, com a participação de 400 atletas representando 23 países ${ }^{4,9}$. Em 1964, foi fundada a Organização Internacional de Esportes para o Deficiente (ISOD), que englobava os amputados, os portadores de deficiência visual, os portadores de Paralisia Cerebral e os portadores de afecções medulares ${ }^{4}$

Em 1975, um atleta norte americano, Bob Hall, participou da maratona de Boston, o que incentivou a participação de outros portadores de deficiência física em corridas de rua. Em 1976, nas Olimpíadas de Toronto, 1500 deficientes competiram representando 36 países. O número de inclusões nas Olimpíadas Especiais aumentou de 1000 para 3200 entre 1967 e $1975^{8}$

Em 1978, formou-se a Associação Internacional de Esportes e Recreação para Paralisados Cerebrais (CP-ISRA) e em 1981, os portadores de deficiência visual formaram a Associação Internacional de Esportes para Cegos (IBSA) ${ }^{4}$.

Desde a primeira Paraolimpíada, os jogos são realizados a cada quatro anos, e na última, em Sidney 2000, participaram 4500 atletas, sendo 64 brasileiros ${ }^{11}$.

O esporte adaptado no Brasil iniciou-se na década de 50, quando os senhores, Robson Sampaio de Almeida, do Rio de Janeiro, e Sérgio Serafim Del Grande, de São Paulo, ficaram deficientes físicos e procuraram serviços de reabilitação nos EUA. Eles fundaram os dois primeiros clubes, o Clube do Otimismo, no Rio de Janeiro, e o Clube dos Paraplégicos, em São Paulo ${ }^{4}$.

Em 1969, o Brasil participou dos II Jogos Pan-americanos em Buenos Aires com sua primeira seleção de basquete em cadeira de rodas, conseguindo a medalha de bronze ${ }^{4}$.

Desde que o País mandou a sua primeira representação aos jogos paraolímpicos, em 1972, na cidade de Heidelberg, na Alemanha, a presença brasileira nestas competições vem se ampliando. Em Sydney, o Brasil teve sua maior delegação em uma Paraolimpíada: 64 atletas, divididos em nove modalidades. O alto nível técnico da delegação enche o Comitê Paraolímpico Brasileiro (CPB) de esperanças quanto a um recorde de medalhas. Na Tabela 1 podemos ver o número de medalhas conquistadas nas Paraolimpíadas em que o Brasil participou ${ }^{11}$.

\begin{tabular}{|l|c|c|c|c|c|c|}
\hline Ano & Local & País & Ouro & Prata & Bronze & Total \\
\hline 1972 & Heildelberg & Alemanha & 0 & 0 & 0 & 0 \\
\hline 1976 & Toronto & Canadá & 0 & 2 & 0 & 2 \\
\hline 1980 & Arnhem & Holanda & 0 & 0 & 0 & 0 \\
\hline 1984 & Nova lorque & EUA & 1 & 3 & 2 & 6 \\
\cline { 2 - 7 } & Stoke Mandeville & Inglaterra & 6 & 14 & 2 & 22 \\
\hline 1988 & Seul & Coreia & 4 & 10 & 13 & 27 \\
\hline 1992 & Barcelona & Espanha & 3 & 0 & 4 & 7 \\
\hline 1996 & Atlanta & EUA & 2 & 6 & 13 & 31 \\
\hline 2000 & Sydney & Australia & 6 & 10 & 6 & 22 \\
\hline \multicolumn{7}{|c|}{ Fonte: $h$ http://www.cpb.org.br/historico.asp } \\
\hline
\end{tabular}

Tabela 1 - Medalhas conquistadas pelo Brasil nas Paraolimpíadas. 
Atualmente, o Esporte Adaptado no Brasil é administrado por 6 grandes instituições: A ABDC (Associação Brasileira de Desporto para Cegos) de deficientes visuais, a ANDE (Associação Nacional de Desporto para Excepcionais) de paralisados cerebrais e dos Les Autres, a ABRADECAR (Associação Brasileira de Desportos em Cadeira de Rodas) que administra as modalidades em cadeira de rodas, a ABDA (Associação Brasileira de Desportos para Amputados) de amputados, a ABDEM (Associação Brasileira de Desportos para Deficientes Mentais) que administra os esportes para deficientes mentais e a CBDS (Confederação Brasileira de Desportos para Surdos) de deficientes auditivos e não está vinculada ao Comitê Paraolímpico Brasileiro (CPB) ${ }^{11}$.

\section{A NATAÇÃO ADAPTADA}

\section{História}

Pouco se tem na literatura sobre a história da natação adaptada. Sabe-se, entretanto, que o uso de exercícios terapêuticos na água é mencionado em obras tão antigas como a de Aureliano, do final do século 5, na qual recomenda natação no mar ou em nascentes quente, e a do médico Jacques Delpech (1777-1838), que escreveu sobre a correção postural com aparelhos e enfatizou o valor da natação para a coluna vertebral ${ }^{12}$.

No final do século 19 e início do século 20, os exercícios aquáticos começaram a ser utilizados como meios corretivos eficientes, e as doenças reumáticas e do aparelho locomotor recebem o tratamento pioneiro nas estâncias termais européias. Mais tarde, novos métodos surgem ressaltando o valor do exercício terapêutico dentro da água, acima do valor de suas características quimiotérmicas. Em 1924, Lowman organiza uma hidroginástica terapêutica, dentro de tanques ou piscinas, para portadores de poliomielite paraplégicos e portadores de outros problemas ortopédicos ${ }^{12}$

A natação é uma das modalidades que participa dos Jogos Paraolímpicos, e o Brasil possui representantes desta modalidade que participam desde 1980, na Holanda. O Brasil já conquistou várias medalhas, inclusive uma de ouro nos Jogos Paraolímpicos de Atlanta, em 1996, através do atleta brasileiro José Afonso Medeiros, nos 50 metros borboleta ${ }^{11}$.

\section{Treinamento}

A iniciação da natação para pessoas com deficiência física normalmente se dá através do Método Halliwick, que ensina desde o controle respiratório até os movimentos básicos de um nado. A partir daí, são utilizadas técnicas de aprendizagem dos nados como na natação normal, claro que respeitando a individualidade e a capacidade de cada pessoa $^{3}$.

método Halliwick foi criado por James McMillan em 1949, na Halliwick School, em Londres. Esse método baseia-se nos princípios científicos da hidrostática, da hidrodinâmica e da mecânica dos corpos, e seu objetivo é de promover todos os aspectos da natação para pessoas com deficiência. As atividades ensinadas pelo método englobam muitas habilidades, como, por exemplo, o aprendizado de como o empuxo e a turbulência afetam o corpo (e como responder a isso), o aprendizado do equilíbrio, as "remadas" e o desenvolvimento dos movimentos de natação básicos ${ }^{3}$.
Os aspectos recreativos da natação, em particular, são muito enfatizados, de modo que as sessões na água não sejam apenas práticas e construtivas, mas também divertidas. Não são utilizados flutuadores ou qualquer ajuda de flutuação artificial. O indivíduo terá que aprender a dar o máximo de seu próprio desenvolvimento e a descobrir como controlar seu equilíbrio natural ${ }^{3}$.

Após a adaptação e a introdução de alguns movimentos básicos dos nados, entra o trabalho das técnicas esportivas dos nados de crawl, costas, peito e borboleta com pequenas concessões à técnica formal. A partir daí, o terapeuta pode utilizar recursos diversos (como pranchas, espaguetes, flutuadores, entre outros) para auxiliar o aprendizado das modalidades dos nados ${ }^{12}$.

\section{Competições}

Desde que as regulamentações dos esportes adaptados foram se estabelecendo, existem classificações para enquadrar as pessoas deficientes em níveis adequados para competições esportivas. Os critérios de classificação obedecem ao grau de deficiência neurológica e de habilidade funcional apresentados ${ }^{12}$

Na modalidade da natação, atualmente a Federação Internacional dos Jogos de Stoke Mandeville (ISMGF) e conseqüentemente a Associação Brasileira de Desporto em Cadeira de Rodas (ABRADECAR), seguem as normas e regulamentos definidos pelo Comitê Paraolímpico Internacional (IPC). Para a competição, são elegíveis todos os portadores de disfunções e incapacidades locomotoras, sendo que a ISMGF e a ABRADECAR ficam com a liberdade para expandir a participação de outros portadores de deficiências locomotoras, que não os lesados medulares, portadores de poliomielite e amputados de MMII, elegíveis em seus estatutos. Esta ampliação de participação deve ser definida em cada evento competitivo, caso contrário, a classificação fica restrita aos atletas elegíveis nos estatutos da ISMGF ${ }^{13}$.

Sistema de Classificação Funcional (FCS) é o nome do sistema utilizado pelo IPC, e baseia-se no potencial residual do atleta e não nas suas limitações motoras. Na natação, o sistema é denominado Sistema de Classificação Funcional da Natação (FCS-SW), utilizando cálculos numéricos para definir a capacidade locomotora. O sistema é expresso em modelos que demonstram a variação na eficácia da propulsão de nadadores portadores de diferentes capacidades locomotoras ${ }^{13}$

A capacidade locomotora do nadador é avaliada no teste de banco. Após este, o mesmo será avaliado na piscina, nadando, o que constitui o teste de água. Toda capacidade locomotora é examinada para: determinação de pontos para o teste da força muscular e/ ou; determinação de pontos para o teste da coordenação e/ ou; determinação de pontos para a mobilidade articular e/ ou; medição do membro amputado e/ ou; medição do tronco ${ }^{13}$.

O nadador sem deficiência motora atinge 300 pontos para as modalidades S e 290 para as modalidades SB.

- Modalidades S, nados: Livre, Costas e Borboleta - braços 130 pontos, pernas 100 pontos, tronco 50 pontos, saída 10 e viradas 10 pontos; 
- Modalidades SB, nado de Peito - braços 110 pontos, pernas 120 pontos, tronco 40 pontos, saída 10 pontos e viradas 10 pontos.

As modalidades S e SB, na ficha de teste, indicam que os pontos devem ser contados para as respectivas modalidades, ou seja, para as do tipo S: nado estilo livre, nado de costas e nado borboleta, a qual possui 10 classes; ou para as do tipo SB: nado de peito, a qual possui 9 classes; ou mesmo para ambas. O déficit mínimo para estar apto a competir é uma perda de 15 pontos ${ }^{13}$.

O procedimento de teste dentro da água vem imediatamente após a determinação dos pontos calculados no teste de banco. Lembrando que o teste dentro da água é, de longe, a parte mais relevante, no processo de classificação ${ }^{13}$.

\section{Benefícios da natação para indivíduos com lesões neurológicas}

O esporte é tão importante para uma pessoa com incapacidade física quanto para um indivíduo saudável. Embora nem todos os indivíduos com incapacidades tenham o desejo de se tornar um atleta, a participação esportiva já traz vários benefícios ${ }^{14}$.

SHEPHARD (1991) citou que a atividade física regular pode trazer novas perspectivas para indivíduos com incapacidades físicas, incluindo novas amizades e até oportunidades de emprego, devido ao aumento da produtividade ${ }^{14}$.

As lesões neurológicas mais comuns em indivíduos jovens são o traumatismo crânio-encefálico (TCE), tendo como principal causa acidente automobilístico ${ }^{15}$; e a lesão medular, sendo que as principais causas são acidente automobilístico, quedas, mergulhos e ferimento por arma de fogo (FAF) ${ }^{16}$. Nas crianças, são seqüelas de paralisia cerebral ${ }^{17}$, e em adultos e outros são o acidente vascular encefálico (AVE) e TCE 15,18. Também encontramos ainda um número significativo de adultos com seqüela de poliomielite ${ }^{19}$.

As seqüelas mais comuns encontradas nos indivíduos portadores de deficiência física são: hemiplegia, paraplegia, tetraplegia, paresias, alterações motoras, espasticidade, alteração de tônus, alteração de equilíbrio e alteração de coordenação 15,16,18,19,20,21

As atividades motoras em meio líquido visam o desenvolvimento cognitivo, afetivo, emocional e social, sendo mencionadas como um excelente meio de execução motora, favorecendo o desenvolvimento global do indivíduo portador de deficiência física 22,23.

Apesar da lesão medular levar a um comprometimento nos sistemas fisiológicos, os problemas psicológicos são os mais freqüentes, tais como raiva, negação, depressão e alterações no humor. Como os exercícios físicos podem reduzir a ansiedade em indivíduos saudáveis, então estes foram sugeridos como uma possível tática para prevenir ou minimizar as perturbações do humor. Indivíduos com lesão medular, geralmente apresentam alterações do humor e exercícios podem melhorar esta alteração, com ganhos na qualidade de vida ${ }^{14,24,25}$

O exercício físico regular pode aumentar o poder aeróbio e a força muscular. A falta de aptidão física em tarefas específicas, por exemplo, pode ser um obstáculo à autonomia do indivíduo e conduzir a uma perda da independência. A prática de exercícios físicos aumenta a capacidade física, proporcionando desempenho menos estressante em tarefas diárias e maior independência funcional ${ }^{26}$

As propriedades físicas da água (densidade, pressão hidrostática, viscosidade, entre outras) irão influenciar no comportamento humano, tanto no aspecto fisiológico como psicológico. Pode-se esperar, então, uma variedade de efeitos. Na musculatura e no aparelho locomotor, ocorrerá uma meIhora na irrigação sangüínea. Com a contração e relaxamento muscular, observada na prática da natação, haverá estímulos necessários para o desenvolvimento da musculatura e conseqüentemente melhora na postura corporal ${ }^{26}$

No coração haverá um fortalecimento da musculatura, bem como um aumento do volume deste. Conseqüentemente, a freqüência cardíaca diminui, a capacidade de transporte de oxigênio aumenta e o esforço cardíaco reduz. Os vasos sangüíneos ficam mais elásticos. Assim, com esta economia de diversas funções circulatórias, o organismo se adapta melhor aos esforços ${ }^{26}$

A natação exige um grande esforço da respiração. Com sua prática, observa-se um incremento da absorção de oxigênio máxima com o aumento do volume de ar que entra para os pulmões através da inspiração mais profunda. Há um aumento da capacidade de difusão do oxigênio e elevação da tolerância relativa ao débito de oxigênio. Além disso, auxilia na prevenção de doenças do aparelho respiratório, assim como do coração e do sistema circulatório ${ }^{26}$. SANTIAGO et. al (1993) e SHEPHARD (1991) referiram que o exercício físico moderado pode diminuir o risco de doenças cardiovasculares e coronarianas em indivíduos com incapacidades físicas ${ }^{14,27}$

Um estudo realizado por CHATARD et. al (1992) avaliou a influência do grau da deficiência em vários aspectos da natação e suas relações com os desempenhos em 100 e 400 metros de nado. Foram estudados 34 nadadores com incapacidades físicas diversas, dos quais 21 eram homens e 13 mulheres. Os nadadores foram divididos em três grupos distintos de acordo com as incapacidades. Este estudo indicou que os desempenhos, a média de consumo de oxigênio e os fatores de impulsão na água foram relacionados com o grau de incapacidade de cada grupo de indivíduos. Confirmou que o consumo de oxigênio e os fatores de impulsão na água são dois grandes determinantes do desempenho de nadadores com incapacidades físicas e nadadores sadios ${ }^{1}$.

Durante o processo de aprendizagem e exercitações dos movimentos impostos pela natação, o sistema nervoso central é chamado para intervir em primeiro lugar, respondendo aos estímulos específicos pela criação de novas reações em reflexos condicionados. Como conseqüência da prática regular da natação, a repetição desses estímulos modifica as estruturas do corpo, advindas, principalmente, da intervenção do sistema nervoso vegetativo ${ }^{26}$.

$\mathrm{Na}$ natação com paraplégicos e tetraplégicos deve-se ter cuidado com relação à falta de sensibilidade que aumenta os riscos de lesões de pele, luxações e fraturas. Para os hemiplégicos, o cuidado deve ser principalmente com relação ao déficit de equilíbrio porque a parte afetada é mais 
pesada, então afunda, modifica rotações e condiciona a escolha do nado a ser praticado. A natação para estes tipos de acometimentos pode promover educação para o lazer; manter a amplitude dos movimentos e conservar a sua função; desenvolver a imagem corporal, procurando a funcionalidade; promover a auto-afirmação que se reflete no relacionamento interpessoal; ajudar a reeducação da bexiga; promover hábitos higiênicos; equilibrar o desenvolvimento exagerado dos membros superiores e propiciar o relaxamento destes grupos musculares solicitados constantemente; equilibrar os desvios da coluna e eixos transversos; estimular as funções cardiovascular e respiratória; e promover a integração social ${ }^{12}$.

A natação pode beneficiar o paciente portador de paralisia cerebral com relação à adequação do tônus acentuado, liberando o potencial de movimento restringido pelos músculos tensos, permitindo a aprendizagem de atividades necessárias para movimentos funcionais através de atividades globais voluntárias e motivantes. Na água a ação da gravidade é quase nula permitindo à criança executar movimen tos que não poderia realizar em solo, o que estimula a propriocepção com a execução de movimentos ou posturas não habituais que auxiliam para a estruturação da imagem corporal. Também proporciona meios de estimulação para o desenvolvimento da fase psicomotora em que se encontra. Além disso, os exercícios de controle respiratórios são importantes para estes pacientes que normalmente possuem alteração da função respiratória ${ }^{12}$

É muito importante salientar que estas atividades físicas esportivas são vitais para indivíduos com seqüelas neurológicas crônicas, que já não participam mais de programas regulares de reabilitação. Esta é a forma ideal de mantê-los em atividade física continuada, prevenindo sua saúde e prevenindo complicações futura e sobretudo, estimulando a sua qualidade de vida e integração social ${ }^{28}$.

Podemos concluir, que o envolvimento do indivíduo portador de deficiência física com a natação traz benefícios não só para sua melhora física como também para seu estado emocional e conseqüentemente melhora de sua qualidade de vida.

\section{REFERÊNCIAS BIBLIOGRÁFICAS}

1. Chatard JC, Lavoie JM, Ottoz H, Randaxhe P, Cazorla G, Lacour JR. Physiological aspects of swimming performance for persons with disabilities. Med Sci Sports Exerc, 24: 1276-1282, 1992

2. Costa MO, Labronici RHDD, Mattos E, Cunha MCB, Oliveira ASB, Gabbai AA. Bocha: uma modalidade esportiva recreacional como método de reabilitação. Rev. Neurociências. 10(1): 24-30, 2002.

3. Association of Swimming Therapy. Natação para deficientes. $2^{a}$ ed. São Paulo. Manole, 2000

4. Labronici RHDD. O esporte como fator de integração do deficiente físico na sociedade. Tese de Mestrado em Neurociências, EPM - UNIFESP, São Paulo, 1997

5. Rimmer JH. Health promotion for people with disabilities: the emerging paradigm shift from disability prevention to prevention of secondary conditions. Phys Ther, 79:495-502, 1999.

6. INDESP. Desporto Adaptado no Brasil: origem, institucionalização e atualidade. Brasília, 1998, pp. 11-140.

7. Jackson RW. Sport for the spinal paralyzed person. Paraplegia, 25: $301-$ 304,1987.

8. Molnar G. Rehabilitative benefits of sports for the handicapped. Connecticut Medicine, 45(9): 574-577, 1981.

9. McCann C. Sports for the disabled: the evolution from rehabilitation to competitive sport. Amer J Sports Med, 30: 279-280, 1996

10. Arbuthnott K. Sport for people with a disability: current state of play. $\mathrm{Br} J$ Sports Med, 32: 275-280, 1998.

11. http://www.cpb.org.br/historico.asp

12. Buckhardt R, Escobar MO. Natação para portadores de deficiências. Rio de Janeiro. Ao Livro Técnico, 1985.

13. http://www.abradecar,org.br/modl/natacao.asp

14. Shephard RJ. Benefits of sport and physical activity for the disabled: implications for the individual and for society. Scand J Rehab Med, 23: 5159, 1991.

15. Rowland LP. Traumatismo. In: Rowland LP (ed.). Merrit Tratado de Neurologia. $9^{a}$ ed. Rio de Janeiro. Gauanabara, 1995. pp. 331-333.
16. Annunthred D, Schneider FJ. Lesão Medular Traumática. In: Annunthred D. (ed.). Fisioterapia Neurológica. São Paulo. Manole, 1994. pp. 421 479 .

17. Gomes C, Santos CA, Silva JUA, Lianza S. Paralisia Cerebral. In: Lianza S (ed.). Medicina de Reabilitação. $2^{\mathrm{a}}$ ed. Rio de Janeiro. Guanabara, 1995. pp. 288-291.

18. Hausen SR, Plotnik R, Castro JC. Acidentes Vasculares Cerebrais. In: Barreto SSM, Vieira SRR, Pinheiro CTS (eds.). Rotinas em terapia intensiva. $3^{a}$ ed. Porto Alegre. Artmed, 2001. pp. 319-320.

19. Battistella LR, Cuzziol LH, Sakamoto H. Síndrome Pós-Pólio: diagnóstico e reabilitação. In: Lianza S (ed.). Medicina de Reabilitação. $2^{a}$ ed. Rio de Janeiro. Guanabara, 1995. pp. 356-357.

20. Bromley I. Tetraplegia and Paraplegia: a guide for physiotherapists. $15^{\mathrm{a}} \mathrm{ed}$. Edinburgh. Churchill Livingstone, 1998.

21. Lianza S, Casalis MEP, Greve JMDA, Eichberg R. A Lesão Medular. In: Lianza S (ed.). Medicina de Reabilitação. $2^{a}$ ed. Rio de Janeiro. Guanabara, 1995. pp. 304-309.

22. Freudenhein AMO. O nadar: uma habilidade motora revisitada. Sao Paulo. EDUSP, 1995

23. Chachan AMA. Reliability and validity of the aquatic adjustment test for children with disabilities. Anais do $13^{\circ}$ International Symposium Adapted Physical Activity. Viena Austria, 2001. p. 24

24. Noreau L, Shephard RJ. Spinal cord injury, exercise and quality of life. Sports Med, 20: 226-250, 1995

25. Jackson RW, Fredrickson A. sports for the physically disabled. Amer J Sports Med, 7: 293-296, 1979

26. Massaud MG, Corrêa CRF. Natação para adultos. Rio de Janeiro. Sprint, 2001.

27. Santiago MC, Coyle CP, Kinney WB. Aerobic Exercise Effect on individuals with physical disabilities. Arch Phys Med Rehab, 74: 1192-1198, 1993.

28. Labronici RHDD, Cunha MCB, Oliveira ASB, Gabbai AA. Esporte como fator de integração do deficiente físico na sociedade. Arq Neuropsiquiatr. 58 (4): $1092-1099,2000$ 